\title{
Correction to: Geometric Tiles and Powers and Limitations of Geometric Hindrance in Self-assembly
}

Daniel Hader and Matthew J. Patitz

\begin{abstract}
Correction to:
Chapter "Geometric Tiles and Powers and Limitations of Geometric Hindrance in Self-assembly" in: I. McQuillan and S. Seki (Eds.): Unconventional Computation and Natural Computation, LNCS 11493, https://doi.org/10.1007/978-3-030-19311-9_16
\end{abstract}

The original version of this paper contained a mistake. Theorem 3 in "Section 4," which claimed that for every temperature-1 system in the Dupled abstract Tile Assembly Model (DaTAM) there exists a temperature-1 system in the Geometric Tile Assembly Model (GTAM) which simulates it at scale factor 1 and using only 2 glues, was incorrect. That theorem and the section that contained it have been removed. Please note that that result was independent of all other results, and the fact that it was incorrect does not impact them or any of the other remaining portions of the paper. 